\title{
Alice in Pheromone Land: An Experimental Setup for the Study of Ant-like Robots
}

\author{
Simon Garnier ${ }^{* a}$, Fabien Tâche ${ }^{b}$, Maud Combe $^{a}$, Anne Grimal ${ }^{a}$ and Guy Theraulaz ${ }^{a}$ \\ ${ }^{a}$ Centre de Recherches sur la Cognition Animale \\ UMR-CNRS 5169 - Université Paul Sabatier \\ Bât 4R3 - 118 Route de Narbonne - F-31062 Toulouse cedex 4 - FRANCE \\ ${ }^{b}$ Autonomous Systems Lab \\ Swiss Federal Institute of Technology Zurich (ETHZ) \\ CLA E18 - Tannenstrasse 3 - CH-8092 Zürich - SWITZERLAND
}

\begin{abstract}
The pheromone trail laying and trail following behaviors of ants have proved to be an efficient mechanism to optimize path selection in natural as well as in artificial networks. Despite this efficiency, this mechanism is under-used in collective robotics because of the chemical nature of pheromones. In this paper we present a new experimental setup which allows to investigate with real robots the properties of a robotics systems using such behaviors. To validate our setup, we present the results of an experiment in which a group of 5 robots has to select between two identical alternatives a path linking two different areas. Moreover, a set of computer simulations provides a more complete exploration of the properties of this system. At last, experimental and simulation results lead us to interesting prediction that will be testable in our setup.
\end{abstract}

\section{INTRODUCTION}

Research in collective robotics is strongly influenced by discoveries made in the last 25 years about the impressive collective abilities demonstrated by social insects [1]. In these animals, natural selection has already shaped many self-organized strategies to efficiently solve problems that are beyond the capabilities of single individuals (e.g. nest-site selection or traffic regulation $[2,3])$.

To reach such refined collective behaviors, mutual communication between agents is often a crucial requirement. This communication can be achieved thanks to physical media, like light and sound, or by chemical ones. Pheromones belong to this latter category. They are chemical signals released by an organism and are available for both direct and indirect communication.

In several ant species, pheromones are well known to be involved in foraging behavior, and more precisely in recruitment of nestmates and navigation between nest and food sources. This is achieved through a simple stigmergic process which results in the formation and reinforcement of a chemical trail linking these different areas. Several experimental and theoretical studies showed that this self-enhanced communication process can lead an ant colony to interesting collective behav-

\footnotetext{
${ }^{*}$ Corresponding author. simon.garnier@cict.fr
}

iors such as the selection of the most rewarding food source [4] or the selection of a single path between the nest and a food source [5] (the shortest one if the alternatives are of unequal length, one of the alternatives at random if they are of equal length).

During the 1990's, a growing number of studies suggested that this pheromone-based process should be an efficient method to solve "human problems": e.g. re-routing traffic in busy telecommunication networks or dealing with the "traveling salesman problem" (finding the shortest route by which to visit a given number of cities, each exactly once) $[6,7]$. These studies were the first proof that the "pheromone logic" can be effectively applied to artificial systems, and therefore to groups of autonomous robots.

But, while a great part of the studies about stigmergic processes in embodied robotics systems focused on object clustering and sorting $[8,9,10]$, only a small part was dedicated to the use of a pheromone-like paradigm. This is partly due to the difficulty to deal with chemical signals in terms of data emission and reception compared to physical ones (but see [11]). Some alternatives to this issue have been proposed: e.g. (1) heat applicators and sensors [12], virtual pheromones stored either by (2) an external computer [13] or by (3) each robot in the group [14], (4) ultraviolet sensitive glowpaint [15]. Solution (1) [12] is not efficient to establish a long-lasting trail. In solution (2) [13], perception of pheromone and control decisions are all performed by an external computer, thus severely limiting the autonomy of the robots. Solution (3) [14], even if really promising in terms of applications, rather resembles to path formation thanks to robot chains as already suggested in [16] than to the stigmergic path formation used by ants. At last, solution (4) firstly developed in an artistic context does not offer a sufficient flexibility for laboratory usage since evaporation of pheromone can not be easily controlled.

In the present work, we propose to use another method to study in laboratory conditions the properties of a robotic system using ant trail laying and trail-following behaviors. We suggest to substitute pheromones with light projected on the 
ground thanks to a video projector as proposed in [17, 18]. This video projector is controlled by a tracking setup which detects robot positions and computes the location and strength of the "light trail" deposit. At last, robots detect and follow light trails thanks to two simple photoreceptors. In order to make the "proof-of-concept" of our setup, we successfully achieved with a group of small autonomous robots Alice [19] the collective selection of a path between a nest zone and a food source zone among two identical possibilities, a well known experiment carried out with ants by Beckers et al. [20]

Of course, this system does not solve the autonomous trail laying problem. However its purpose is not to become a real life application but rather to provide a cheap and very easy to handle laboratory tool to test pheromone algorithms with robots that perceive their environment and adapt their behavior in a fully autonomous way.

This paper is divided into 3 sections. In section II, we describe the experimental setup and the behavioral model used in this study. In section III on page 4, we present our first experimental results in a path selection paradigm as well as a more complete exploration of the model with simulations. At last, in section IV on page 6, we discuss some interesting opportunities offered by our experimental setup.

\section{MATERIALS AND METHODS}

\section{A. Robot Alice}

\section{Base robot}

The micro-robots Alice were designed at the EPFL (Lausanne, Switzerland, see the base robot on the right of Fig. 1) [19]. They are very small robots $(22 \mathrm{~mm} \times 21 \mathrm{~mm} \times 20 \mathrm{~mm})$ with a maximum speed of $40 \mathrm{~mm} \mathrm{~s}^{-1}$. They are equipped with two watch motors with wheels and tires. Four infrared (IR) sensors and transmitters are used for communication and obstacle detection. Energy is provided by a NiMH rechargeable battery allowing an autonomy of about 3.5 hours in our experimental conditions. The robots have a microcontroller PIC16LF877 with 8K Flash EPROM memory, 368 bytes RAM and no builtin float operations. Programming is done with the IDE of the CCS-C compiler allowing to use assembler and C commands at the same time, and the compiled programs are downloaded in the Alice memory with the PIC-downloader software ${ }^{1}$.

\section{Trail following add-on}

An add-on module has been built to allow light path detection by the robots and robot detection by a tracking device. This module is plugged into the top connector of the Alice robot, as can be seen on the left of Fig. 1. This add-on is equipped with two photodiodes pointing upwards which let the robot detect the trail. It also carries a red LED (Light Emitting Diode) to permit an easy and reliable tracking in conditions of changing

\footnotetext{
${ }^{1}$ http://www.ehl.cz/pic
}

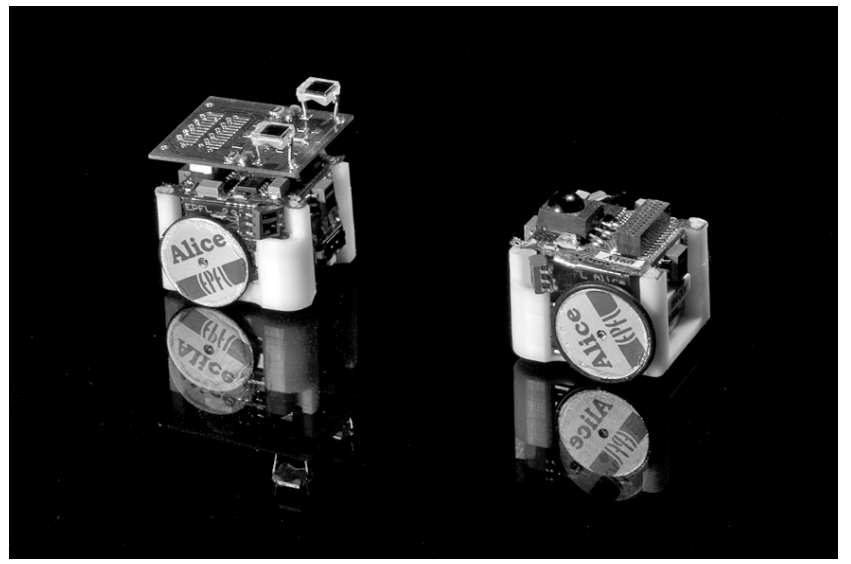

Figure 1. Robot Alice with (left) and without (right) the additional module for light detection.

background brightness. Additionally, the LED provides a very simple solution to indicate to the tracking device the robot's state: with the LED turned on, the robot does lay pheromones and thus must be video-tracked, with the LED turned off the robot is only exploring without trail laying and no tracking is necessary. Technical details about this add-on can be found in [18].

\section{B. Experimental setup}

The experimental setup has three parts: a diamond shaped maze, a robot tracking device and a pheromone deposit device. The whole setup is held by a $2 \mathrm{~m} \times 1.5 \mathrm{~m} \times 3 \mathrm{~m}$ aluminium cage with three opaque walls to avoid robots or tracking device being disturbed by external light. The fourth wall is left open and points towards a direction with no light source. All experiments are videotaped with a Sony 3CCD DCRTRV950E video camera.

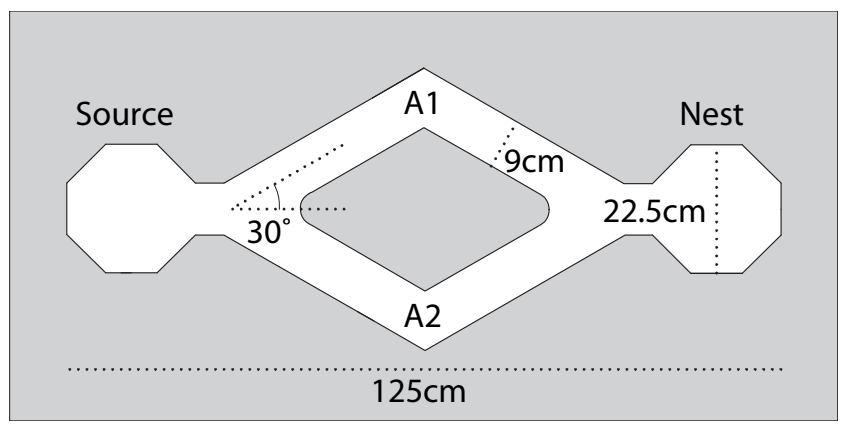

Figure 2. Blueprint of the experimental setup.

\section{Maze}

The maze is built with white cardboard ( $5 \mathrm{~mm}$ thick) according to the blueprint in Fig. 2 (wall height of $2.5 \mathrm{~cm}$ ). It lies on the ground of the cage. Each extremity of the maze is an octagonal 
area which represents either the nest or the source. In each of these areas, two infrared transmitters built into the walls continuously emit a signal (different for each area) which allows the robots to know if they are in the nest or the source. Nest and source are linked by a diamond shape maze with two arms (A1 and A2) of the same length deviating from each other by a $60^{\circ}$ angle.

\section{Tracking device}

The goal of the tracking device is to detect the red LED on the top of each trail laying robot. The tracking device is made up with a firewire digital video camera Unibrain Fire-i400 (resolution $640 \times 480$ ) hung about $1.5 \mathrm{~m}$ above the maze and connected to a laptop computer Dell Latitude D810 thanks to a 1394a PCMCIA card. Image acquisition is done with the open source CMU 1394 Digital Camera Driver (Robotics Institute, Carnegie Mellon University ${ }^{2}$ ) and image treatment is done with the open source OpenCV library $\left(\mathrm{Intel}^{3}\right)$.

Usually a picture is stored with the three channels RGB (Red, Green, Blue). If one would just look at the red channel, he could see the robot's LED as a bright spot, but also all virtual pheromone trails. Additionally, the red portion of the LED changes if the robot is in a dark or in a bright area. A better way for detection is to calculate the HSV channels (Hue, Saturation and Value) from the RGB channels. The resulting $\mathrm{H}$ value is the angle in a color circle. If the red color does get brighter or darker, the $\mathrm{H}$ value will stay the same. Once the $\mathrm{H}$-channel is extracted, white noise is removed thanks to morphological opening (erosion followed by dilatation) with a $3 \times 3$ matrix. Then a maximum and minimum threshold are applied to turn the resulting image into a binary one and a fit ellipse function returns the centre positions of the robots.

The described tracking function has proven to be very stable. The HSV decomposition is a reliable way to track the red spot. Even with room light turned on, or bad camera settings, the program can still track the robots in most situations.

\section{Pheromone deposit device}

Once the position of a robot emitting pheromones is known, light has to be sent to this location. An output image $(800 \mathrm{x}$ 600 pixels) with luminous trails is produced and displayed in a window. This window is running in full screen mode on the enhanced desktop of Windows XP the video projector (Sony VPL-CX5) is connected to. To obtain a sufficiently large image to cover the whole maze, the video projector is hung $3 \mathrm{~m}$ above it. The image is composed with uniformly blue spots (blue is chosen to contrast with the red LED of robots), each of them centred on the successive positions of robots, but without overlapping between the successive spot of a given robot. The light intensity of the blue spot is used to simulate the intensity of the pheromone deposit. Positions of pheromone spots are also corrected to take into account camera lens distortion

\footnotetext{
${ }^{2}$ http://www.cs.cmu.edu/ iwan/1394/

${ }^{3} \mathrm{http}: / /$ sourceforge.net/projects/opencvlibrary/
}

(thanks to the Camera Calibration Toolbox for Matlab ${ }^{4}$ ) and misaligning of the tracking camera and the trail laying video projector. Each point has a $6 \mathrm{~cm}$ diameter. This diameter was chosen to allow two robots to cross each other and thus to reduce traffic jam on the trail.

At last, if no other deposit is done at a given point, light intensity $(I)$ decreases following an exponential decay to simulate pheromone evaporation:

$$
I(t)=I(t-\Delta t) \exp \left(\left(\log (1 / 2) / t_{c}\right) \Delta t\right)
$$

With $t$, the current time, $\Delta t$, the period between two evaporation time-steps and $t_{c}$ the characteristic evaporation time. To lower the processing charge (the previous computation is applied to each pixel in the image), evaporation is triggered every 5 seconds. All treatments included, the tracking and trail laying software allows an effective speed of about 5 images per second. This is sufficient for our needs.

\section{Behavioral model}

The behavioral model is a generic and simplified model of trail laying and trail-following behaviors in ants. It aims at capturing the essential features needed to achieve a path selection "as ants do".

In the absence of light pheromones, a robot (laying a trail or not) moves according to a correlated random walk, with a strong tendency to continue in the same general direction. This behavior is called "exploratory behavior". If the robot detects an obstacle, it tries to avoid it by turning in the opposite direction. This behavior is called "avoidance behavior". If the robots detects a luminous trail with its photoreceptors, it tries to turn towards the one receiving more light. This behavior is called "trail following behavior" (see figure 5 on page 5).

Each of these behaviors triggers the computation of a movement vector. The three vectors are summed together with different weights to obtain the new direction at each time step (50ms). The exploratory vector points ahead of the robot and changes randomly between $-90^{\circ}$ and $90^{\circ}$ after a time drawn in a decreasing exponential distribution. The avoidance vector is the sum of four vectors, each of them pointing in the opposite direction of one of the four proximity IR sensors of the robot. Their intensity grows with the intensity of the signal received by their respective sensor. At last, the trail following vector aims either to the right or the left of the robot. Its direction and intensity are controlled by the difference between light intensities perceived by the right and the left photoreceptor.

The trail laying behavior of the robot is controlled as follows. The robot begins to lay pheromone (i.e. to switch on its red LED) only when it leaves the source area (i.e. when it loses the IR source signal, see section 1 on the preceding page of section $\mathrm{B}$ on the previous page). It then stops trail laying (i.e. switches off the red LED) when it enters either the nest

\footnotetext{
${ }^{4}$ http://www.vision.caltech.edu/bouguetj/calib_doc/
} 

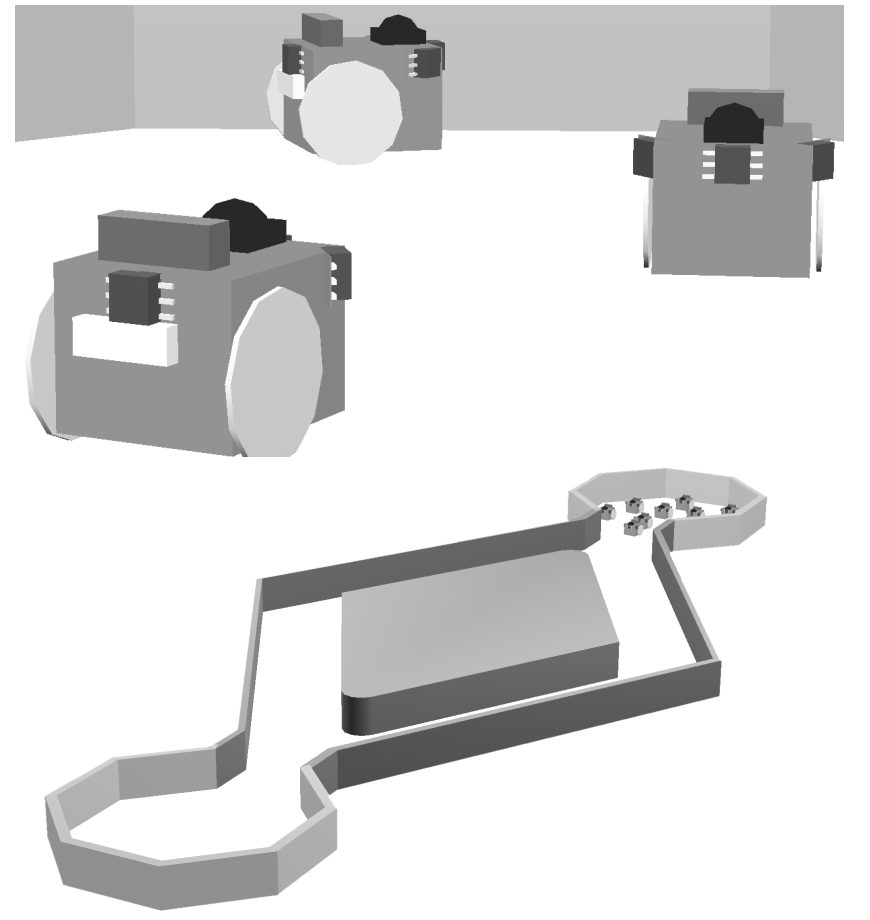

Figure 3. Pictures of the simulations in Webots. Top: three simulated robots Alice. Bottom: overview of the simulated setup.

or the source area (i.e. when it detects the IR nest or source signals).

\section{Simulations}

Before any experiment, a set of 8100 simulations was done with the Webots software (version 5.1.9) with physics engine switched on [21] on a Power Mac G5 2x2.3 GHz with Mac OS $\mathrm{X}$ 10.4.8. In the simulations, pheromone deposits were gray spots laid on the ground by the simulated robots. The gray level $(0=$ white, $1=$ black $)$ was chosen to represent the intensity of the pheromone deposit. Simulated robots followed the pheromone trail thanks to light sensors installed under their body.

Simulations were used to assess the influence of three parameters of the model: the number of robots $(1,2,3,5$ and 10), the intensity of the pheromone deposit (six different intensities were tested between 0.03 and 0.5 ) and the characteristic evaporation time $t_{c}$ (see above, 9 different times were tested, varied between 60 and 3600 seconds). For each combination of parameters, 30 simulation runs were done. They were intended to estimate the parameters to use in experiments with real robots so as to obtain stable decisions.

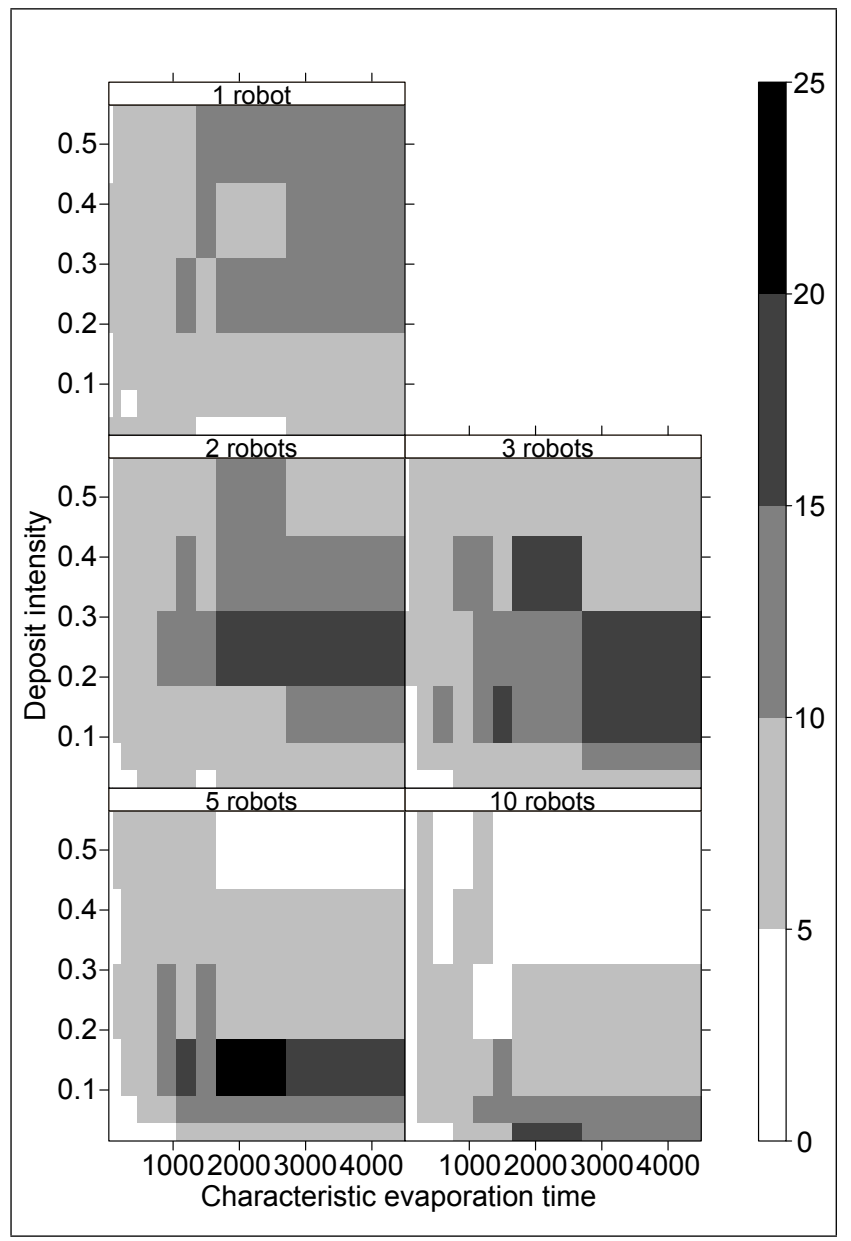

Figure 4. Mean duration of a choice event (gray levels, in minutes) as a function of the intensity of the pheromone deposit, the characteristic evaporation time $t_{c}$ and the number of robots.

\section{RESULTS}

This section is divided in three parts. After a brief description of the calculation of the probability to choose each arm of the maze, we present the exploration with simulations of our robotics model followed by our first experimental results obtained with robots Alice. We show results of ten experiments with one robot alone and ten experiments with five robots. Simulations and experiments cover a real time period of 60 minutes.

\section{A. Data analysis}

For each minute of each simulation and experimental run, we observed the number of robots coming from the source and entering in the arms $\mathrm{A} 1\left(n_{A 1}\right)$ and $\mathrm{A} 2\left(n_{A 2}\right)$. We then computed the proportion $P_{i}$ of robots entering arm A1 over a sliding time window of ten minutes. That is, for each minute $i \in[0: 50]$, 
we computed:

$$
P_{i}=\frac{\sum_{i \rightarrow i+10}\left(n_{A 1}\right)}{\sum_{i \rightarrow i+10}\left(n_{A 1}+n_{A 2}\right)}
$$

We thus obtain the temporal dynamics of the probability for the robots to choose each arm of the maze (for an example of this dynamics, see Fig. 6-A). At the beginning of the experiment $(i=0), P_{0}$ was set to 0.5 .

\section{B. Simulation results}

In order to evaluate the efficiency of each combination of parameters we must defined a criterion representative of a stable choice. We first defined a choice event each time the probability $P_{i}$ becomes superior to 0.75 (A1 chosen) or inferior to 0.25 (A2 chosen). For each simulation, we counted the number and the duration of these choice events. For each set of parameters, we computed the mean duration of choice events. This mean duration is a good indicator of a stable choice: if its value is low, it means that either no choice was made (the probability stays between 0.25 and 0.75 ) or the choice was not stable (many choice events of short duration). A high value indicates a strong and stable choice. The results for our simulations are shown in Fig. 4.

This figure clearly illustrates the three following points:

- Whatever the number of robots, the highest mean duration values occur only with long characteristic evaporation times (1200 to 3600 seconds). If evaporation is too fast, no stable choice can take place.

- When the number of robots grows, the pheromone deposit intensity needed to obtain a stable choice decreases. In other words, the individual cost of pheromone production decreases with the size of the group.

- At last, the highest mean duration (i.e. the maximum in each plot) grows with the number of robots, reach a maximum with 5 robots and then drop for 10 robots. This is mainly due to the saturation in pheromone of both arms of the maze that occurs more frequently (but not every time) when the number of robots grows.

The same analysis was done for robots coming from the nest. Because no differences with robots coming from the source were found, these results are not shown.

\section{Experimental results}

A picture of an experiment with robots Alice following a trail is shown in Fig. 5. To obtain the best experimental results according to the simulation data, we chose to work with a group of 5 robots, a deposit intensity of 0.12 and a characteristic evaporation time of 1800 seconds. However, a first set of experiments showed that these parameters in our experimental setup led the

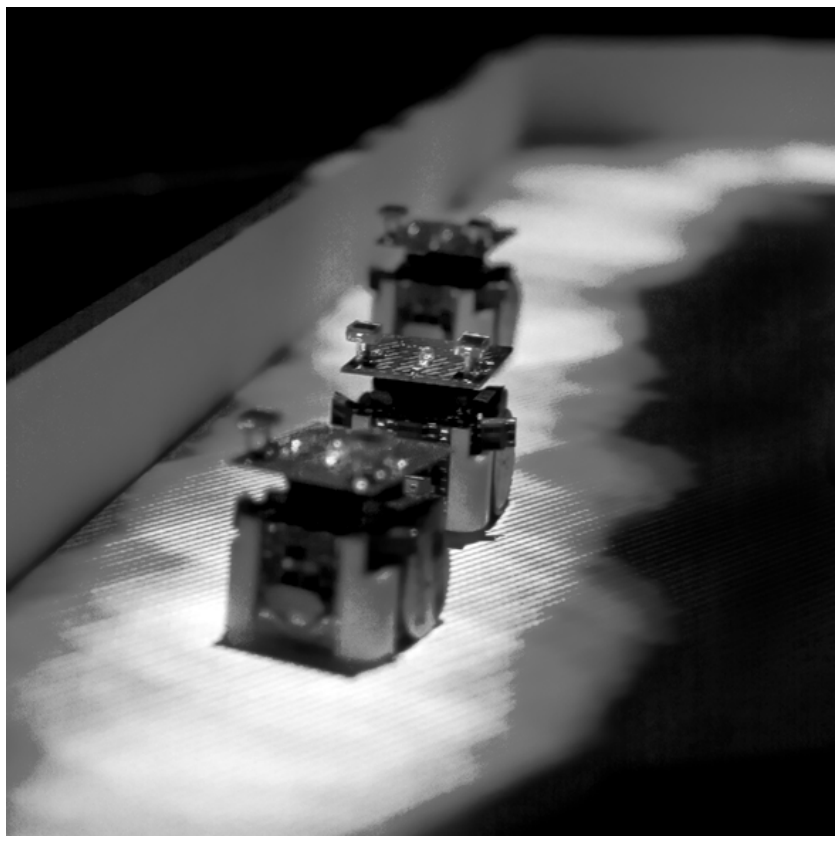

Figure 5. Three robots Alice pursuing a luminous trail.

system to a pheromone saturation of the whole maze. The reason for this problem is the following. The intensity of pheromone deposit (in simulations and experiments) varies between 0 and 1 according to a scale with 256 steps (256 gray levels in simulations, 256 blue levels in experiments). But the dynamic range, i.e. the number of undertones of a given color, our video projector is able to display is below this number. Therefore, the luminous trail intensity grows faster in experiments than in simulations. To counterbalance this effect, we lowered in experiments the characteristic evaporation time to 600 and the intensity of the pheromone deposit to 0.06 . We used the same parameters in experiments with one robot.

For each experiment (one and five robots), we computed in each direction (from the nest, forward; from the source, backward) $P_{i}$ as described above. We represented $P_{i}$ as a function of the time for these four conditions in Fig. 6-B.

This figure clearly shows that for one robot and whatever the moving direction, the probability $P_{i}$ stays around 0.5 and rarely goes up 0.75 or down 0.25 . Therefore we can conclude that no choice happens in these experiments and we can consider them as a kind of "no choice" control.

For five robots, the situation is very different. In most of the experiments, $P_{i}$ quickly overcomes either the high $(0.75)$ or the low (0.25) choice threshold, and then remains beyond these limits. $9 / 10$ of the experiments in the forward direction and $7 / 10$ in the backward direction ended with a clear choice for one of the two arms. The difference between forward and backward runs is not significative $\left(\chi^{2}=1.25, \mathrm{p}=0.2636\right)$. 


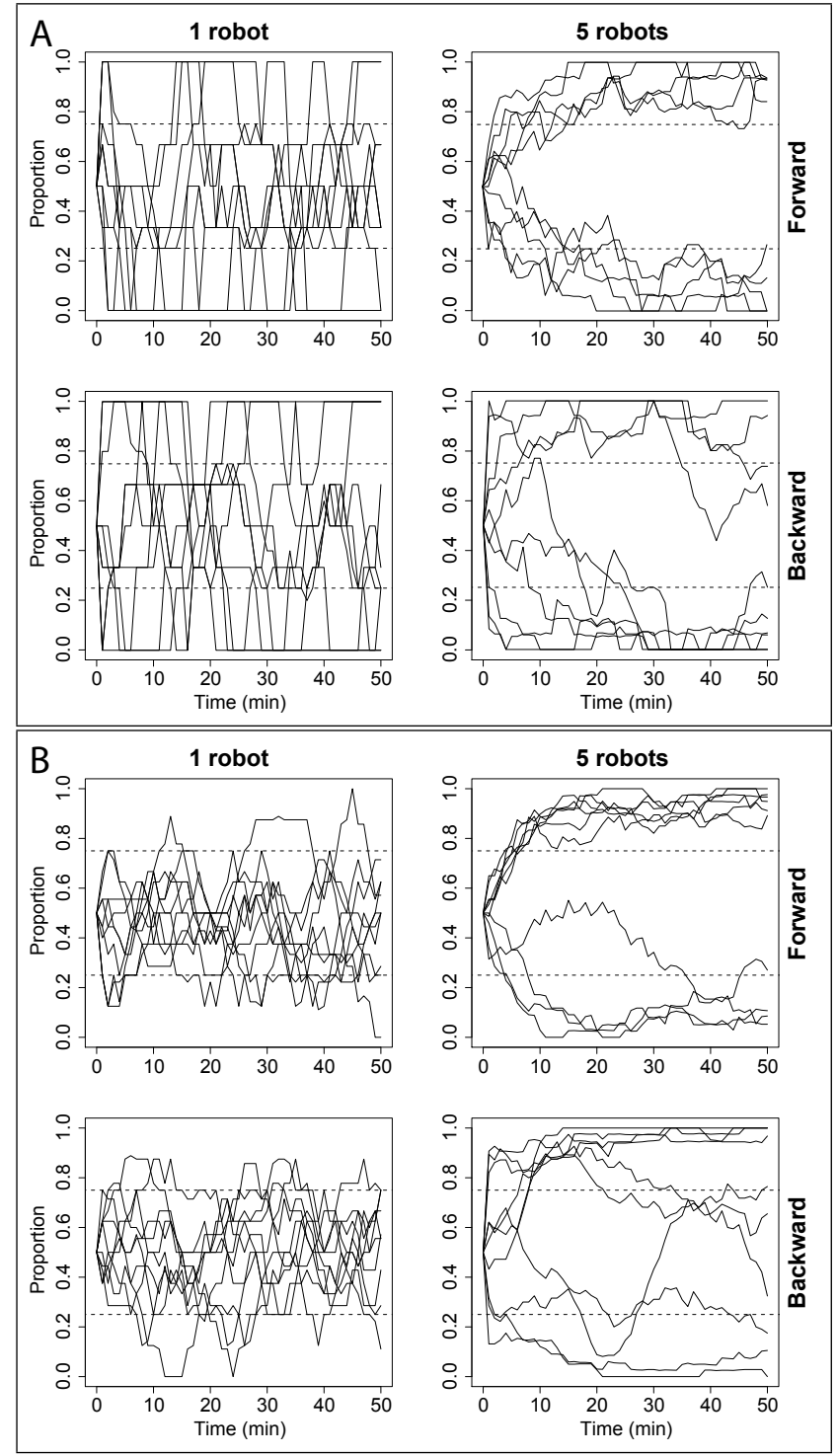

Figure 6. Temporal dynamics of the probability $P_{i}$ to choose the arm A1. Box A: Simulations. Box B: Experiments. In each box: Left, 10 repeats with one robot; Right, 10 repeats with five robots; Top, robots are moving from the nest to the source (forward); Bottom, robots are moving from the source to the nest (backward).

\section{DISCUSSION}

In this paper, we presented a cheap and easy to handle experimental setup to test in laboratory conditions the applicability of the trail laying and trail following behaviors of ants to control a group of small autonomous robots. The proof of concept was done thanks to a very simple experiment in which a group of robots has to choose between two identical paths that link their nest to a food source. Our results show that a group of 5 robots is able to efficiently solve this task, simply following a very simple and generic model of trail laying and trail following behaviors inspired by research about ant foraging.

This paper also presents results of computer simulations that provide a more detailed description of the properties of this robotics system. It appears that in such a system, a collective choice occurs only if the speed of pheromone evaporation is not too fast. Results also suggest that an optimal number of robots is required to get a quick and stable collective choice. At last, it seems that while the number of robots in the system grows, the quantity of pheromone needed to obtain a choice decreases: the individual cost of laying pheromone decreases with the size of the group.

Such an exploration of system's parameters (whatever the method used, simulations, artificial evolution, etc) is useful to estimate the combination of factors that would give the best results in real experiments and avoid losing too much time finding them experimentally. It is also useful to gain some insight about the way the system behaves. However, at least in the context of biological based robotics, we think that experimental validation is the best proof that a given control algorithm works as it was hypothetized [22,23] and that simulation only remains a representation of the reality with all its lacks and simplifications.

Now that the feasibility of the project is established, we can consider a more systematic study of the collective properties of our system and of their control. The experimental paradigm and the behavioral model used in this work, although well adapted to illustrate our intentions, are very simple. They do not bring new results to the study of collective decisions or swarm intelligent systems. Of course that was not the purpose of this article which is rather a description and a validation of our experimental setup. But now we can ask more interesting questions and test them with our setup. In particular, what would happen in more complex situations? How would the system deal with more elaborate networks?

It is now known that artificial agents [7] as well as real ants $[20,24]$ are able to face networks with more than two alternatives and/or with alternatives of dissimilar qualities. One of our current challenge is now to test pheromone based controller in two additional setups: the first one, with two arms of different lengths, to test whether the robots are able to collectively choose the shortest one as ants do; the second one, with several choice points (similar as the one used with ants by Vittori et al. [24]), to test whether the robots are able to deal with complex networks, how they manage their traffic between several possible routes and how they redistribute their traffic if jamming appears. Potential applications of such controllers can be found in adaptative car traffic management or network exploration and exploitation by groups of robots (sewer, piping, landmine, etc).

Another promising challenge would be to provide some control algorithms such that the group of robots is able to adapt its behavior to changing environmental conditions in a fully au- 
tonomous way. One of the simulation results is that it exists an optimal number of robots that allows a quick and stable collective choice. This result is consistent with other works about self-organized collective behaviors in robotics $[25,26]$. We believe that this optimal number strongly depends on the configuration of the experimental setup: length of path between nest and source, width of arms of the maze, etc. And in an unknown environment, it would be hard to estimate this optimal number. Thus, the group of robots should dynamically adapt its size to remain efficient.

To increase group size, robots coming back from the source should recruit other robots. This could be easily done by letting these robots emit a signal (I.R. signal for instance) stimulating robots in the nest to start moving. Here again, recruitment processes used by ants could be used as a source of inspiration. For instance, it was showed in [25] that an ant-inspired tandem recruitment increase the foraging efficiency of groups of 3 to 12 robots.

Recruitment processes should also be counterbalanced by mechanisms able to reduce group size. This is necessary to avoid the system to overshoot the optimal group size, and thus to become less efficient. These mechanisms could act on different parts of robot behavior: robots could stop laying pheromone or reduce the quantity of deposited pheromone; they could stop recruiting; or they could stop foraging. But they should all rely on a less intuitive mechanism able to evaluate the number of robots currently involved in the task. The most promising way to evaluate such a number could again come from the ants. Indeed, ants are able to evaluate the density of their nestmates thanks to the rate of antennae contacts between them and to use this information to regulate their traffic organisation $[27,28,3]$. Such contacts between robots could then be used to estimate the local density of agents. And then, if this density goes above a threshold value, trigger one or several of the group size limiting behaviors mentioned above.

If control algorithm can be easily tested with our setup, it is conspicuous that it remains a tool for laboratory studies and not for real life applications. Nevertheless we are confident that an appliable solution to the problem of pheromone laying and sensing will be soon available. Several leads are promising concerning robot navigation in human constructions. For instance the use of UV sensitive glowpaint [15] (this paint emits green brightness after being stimulated by a UV emitter carried by a robot), if not practical for laboratory studies, becomes an interesting alternative for usage in urban networks as sewer or waterway. One can also consider the use of RFID dispersed in everiday environment [29] that could act store pheromone deposit as discretisized spots. However robots are not really autonomous with these alternatives. Indeed, in unknown environments the problem is more challenging and requires robots able to lay and to sense pheromone by themselves. And at our knowledge no satisfactory alternative has been suggested by now other than the use of chemical markers and sensors [11].
To conclude this paper, we would like to emphasize the fact that the "pheromone logic" provides very interesting opportunities in terms of control algorithms for groups of autonomous robots. And most of these opportunities can now be tested with real robots thanks to our "light pheromone" trail laying setup.

\section{Acknowledgement}

We would like to thank Nikolaus Correll for designing the Webots model of the robot Alice, Olivier Michel for his help in programming our simulator and Jacques Gautrais for his helpful advices. Many thanks to all ASL members for their nice welcome and their technical help. Simon Garnier is partly funded by an ATUPS grant of the University Paul Sabatier.

\section{REFERENCES}

[1] S. Camazine, J.-L. Deneubourg, N. R. Franks, J. Sneyd, G. Theraulaz, and E. Bonabeau, Self-organization in biological systems. Princeton: Princeton University Press, 2001.

[2] R. Jeanson, J.-L. Deneubourg, A. Grimal, and G. Theraulaz, "Modulation of individual behavior and collective decisionmaking during aggregation site selection by the ant Messor barbarus," Behavioral Ecology and Sociobiology, vol. 55, pp. 388394, 2004.

[3] A. Dussutour, V. Fourcassié, D. Helbing, and J.-L. Deneubourg, "Optimal traffic organization in ants under crowded conditions," Nature, vol. 428, no. 6978, pp. 70-3, 2004.

[4] S. Goss, S. Aron, J.-L. Deneubourg, and J. M. Pasteels, "Selforganized shortcuts in the argentine ant," Naturwissenschaften, vol. 76, pp. 579-581, 1989.

[5] J.-L. Deneubourg and S. Goss, "Collective patterns and decision making," Ethology Ecology and Evolution, vol. 1, pp. 295-311, 1989.

[6] E. Bonabeau, M. Dorigo, and G. Theraulaz, Swarm intelligence : from natural to artificial systems. Oxford: Oxford University Press, 1999.

[7] M. Dorigo, E. Bonabeau, and G. Theraulaz, "Ant algorithms and stigmergy," Future Generation Computer Systems, vol. 16, no. 8, pp. 851-871, 2000.

[8] R. Beckers, O. E. Holland, and J.-L. Deneubourg, "From local actions to global tasks: stigmergy and collective robotics," in Proceedings of the Fourth Workshop on Artificial Life (R. Brooks and P. Maes, eds.), (Cambridge, MA), pp. 181-189, MIT Press, 1994.

[9] O. E. Holland and C. Melhuish, "Stigmergy, self-organisation, and sorting in collective robotics," Artificial Life, vol. 5, pp. 173-202, 1999.

[10] A. Martinoli, A. J. Jispeert, and F. Mondada, "Understanding collective aggregation mechanisms: from probabilistic modelling to experiments with real robots," Robotics and Autonomous Systems, vol. 29, pp. 51-63, 1999.

[11] R. A. Russell, "Ant trails - an example for robots to follow?," in Robotics and Automation, 1999. Proceedings. 1999 IEEE International Conference on, vol. 4, pp. 2698-2703, 1999. 
[12] R. A. Russell, "Heat trails as short-lived navigational markers for mobile robots," Robotics and Automation, vol. 4, pp. 35343539, 1997.

[13] J. L. Pearce, P. E. Rybski, S. A. Stoeter, and N. Papanikolopoulos, "Dispersion behaviors for a team of multiple miniature robots," in Proceedings of IEEE International Conference on Robotics and Automation, vol. 1, pp. 1158-1163, 2003.

[14] D. Payton, R. Estkowski, and M. Howard, "Pheromone robotics and the logic of virtual pheromones," in Swarm Robotics WS 2004, vol. 3342 of Lecture Notes in Computer Science, pp. 4557, Jan. 2005.

[15] M. Blow, "'stigmergy': Biologically-inspired robotic art," in Proceedings of the Symposium on Robotics, Mechatronics and Animatronics in the Creative and Entertainment Industries and Arts, The Society for the Study of Artificial Intelligence and the Simulation of Behaviour, 2005.

[16] S. Goss and J.-L. Deneubourg, "Harvesting by a group of robots," in Toward a Practice of Autonomous Systems, Proceedings of the First European Conference on Artificial Life (F. J. Varela and P. Bourguine, eds.), (Cambridge, Massachussets, Cambridge, England), pp. 195-2054, The MIT Press, 1991.

[17] K. Sugawara, T. Kazama, and T. Watanabe, "Foraging behavior of interacting robots with virtual pheromone," in Proceedings of the IEEE/RSJ International Conference on Intelligent Robots and Systems, vol. 3, pp. 3074-3079, 28 Sept.-2 Oct. 2004.

[18] P. Siegrist, "Simulation of ant's pheromone deposition with alice robot," tech. rep., École Polytechnique Fédérale de Lausanne, February 2005.

[19] G. Caprari, T. Estier, and R. Siegwart, "Fascination of down scaling - Alice the sugar cube robot," Journal of Micromechatronics, vol. 1, no. 3, pp. 177-189, 2002.

[20] R. Beckers, J.-L. Deneubourg, S. Goss, and J. M. Pasteels, "Collective decision making through food recruitment," Insectes Sociaux, vol. 37, pp. 258-267, 1990.

[21] O. Michel, "Webots: symbiosis between virtual and real mobile robots," in Virtual World 98, LNAI 1434, (Berlin, Heidelberg), pp. 254-263, Springer-Verlag, 1998.

[22] B. Webb, "What does robotics offer animal behaviour?," Animal Behaviour, vol. 60, no. 5, pp. 545-558, 2000.

[23] B. Webb, "Can robots make good models of biological behaviour?," Behavioral and Brain Sciences, vol. 24, pp. 1033-50; discussion 1050-94, Dec 2001.

[24] K. Vittori, G. Talbot, J. Gautrais, V. Fourcassié, A. F. R. Araujo, and G. Theraulaz, "Path efficiency of ant foraging trails in an artificial network," Journal of Theoretical Biology, vol. 239, pp. 507-515, 2006.

[25] M. J. B. Krieger, J.-B. Billeter, and L. Keller, "Ant-like task allocation and recruitment in cooperative robots," Nature, vol. 406, no. 6799, pp. 992-995, 2000.

[26] W. Agassounon and A. Martinoli, "A macroscopic model of an aggregation experiment using embodied agents in groups of time-varying sizes," in Proceedings of the 2002 IEEE Systems, Man and Cybernetics Conference, (Hammamet, Tunisia), IEEE Press, 2002.

[27] D. M. Gordon, R. E. Paul, and K. Thorpe, "What is the function of encounter patterns in ant colonies?," Animal Behaviour, vol. 45, no. 6, pp. 1083-1100, 1993.
[28] M. Burd and N. Aranwela, "Head-on encounter rates and walking speed of foragers in leaf-cutting ant traffic," Insectes Sociaux, vol. 50, no. 1, pp. 3-8, 2003.

[29] M. Mamei and F. Zambonelli, "Spreading pheromones in everyday environments through rfid technology," in Proceedings of the 2nd IEEE Swarm Intelligence Symposium, (Pasadena, California, USA), 8-10 june 20052005. 